\title{
Analysis of Risks and Strategies of Investment in Global Sports Industry
}

\author{
Yuhao Wang ${ }^{1, *}$ \\ ${ }^{1}$ University of Southampton, Faculty of Social Sciences, Southampton, UK \\ *Corresponding author. Email: yw11a19@soton.ac.uk
}

\begin{abstract}
Nowadays, people all over the world are paying more attention to sports, like NBA's games broadcasting to the media platforms in various countries. There are some phenomena, such as the quantities of people who participate in sports games, and the cost for sport increases dramatically, with both individuals and enterprises involving in sport market. In order to gain benefits, they are willing to invest the sport industry. For instance, people are tending to buy stocks, join the game of guessing sports lottery, and hold shares in the sport company. In this article, the author will start at the analysis of investment in sport industry by using some relevant modules, and analyzing real persuasive example in countries with a large population base. The main target of this article is to analyze some basic strategies in investment of sports, including the government's policies, following up the sports events and not directly investing in sport market. In conclusion, the author suggests that it is a sensible time to invest in sports industry, and due to the expansion of the sports market, the number of investable projects has also increased.
\end{abstract}

Keywords: Risks, Investment strategy, Sports industry, Sports market.

\section{INTRODUCTION}

During 2006 to 2015 , the global sports market is on an upward trend in terms of revenue. "Figure 1" and "Figure 2" illustrate the annual revenue from the sports market. In the intervening decade or so, sports market revenues have increased $44.4 \%$, which is an amazing phenomenon. The media sector, which is closely linked to the sports industry, has also seen significant expansion. According to a report, published by $\mathrm{PwC}$, there are three dominant modules in the sports market, including gate, sponsorship and media right, which account for $32.6 \%, 28.8 \%$ and $24.1 \%$ respectively. The purpose of this article is to provide investors some advice by analyzing the risks that can be encountered in sports investments, and to understand the characteristics of these risks for being able to prevent them. An investment strategy is constructed by involving the factors that need to be taken into account when investing in the sports industry. To provide investors with a clearer direction and objectives through an investment strategy, the analysis in the article will start from two main points. First, the economic performance will be considered, which is a special and direct module to evaluate the benefits of investment, involving real conditions. In this part, the main method will focus on the people who buy the stock in sport industry, and the author will illustrate how they get profit easily in some special sport market. Moreover, the article will demonstrate the risks of investment. Second, the risks of investment activities will be listed and analyzed.

\begin{tabular}{|c|c|c|c|c|c|c|c|c|c|c|c|}
\hline Component & 2006 & 2007 & 2008 & 2009 & 2010p & 2011 & 2012 & 2013 & 2014 & 2015 & $\begin{array}{r}\text { CAGR } \\
2011-15\end{array}$ \\
\hline Global revenues & 107,516 & 111,934 & 120,760 & 112,489 & 121,391 & 118,690 & 129,929 & 130,164 & 146,469 & 145,341 & \\
\hline$\%$ Change & 12.1 & 4.1 & 7.9 & (6.8) & 7.9 & (2.2) & 9.5 & 0.2 & 12.5 & $(0.8)$ & 3.7 \\
\hline
\end{tabular}

Figure 1 Global revenues 2006-2015 [5]. 


\begin{tabular}{|c|c|c|c|c|c|c|c|c|c|c|c|}
\hline Component & 2006 & 2007 & 2008 & 2009 & 2010p & 2011 & 2012 & 2013 & 2014 & 2015 & $\begin{array}{r}\text { CAGR } \\
2011-15\end{array}$ \\
\hline Media rights & 24,281 & 23,861 & 27,005 & 24,563 & 29,225 & 26,945 & 32,141 & 30,062 & 37,801 & 35,247 & \\
\hline$\%$ Change & 19.9 & $(1.7)$ & 13.2 & $(9.0)$ & 19.0 & $(7.8)$ & 19.3 & (6.5) & 25.7 & (6.8) & 3.8 \\
\hline
\end{tabular}

Figure 2 Media rights 2010-2015 [5].

\section{OVERVIEW OF SPORT INDUSTRY}

Sport industry becomes more and more popular and the numbers of citizen who participate in some global sport projects increased dramatically. As the "Figure 3" shows that the size of China's sports market is expanding gradually. According a report, amounts of companies are achieving their global goals through the internationalization of competitive sports. With the development of science and technology, especially the development of satellite broadcasting technology, spectators all over the world can see various exciting sports competitions hold all over the world through TV and the Internet. Sports are becoming more and more influential among viewers around the world [1]. For example, the authority of NBA's television relay has been purchased by media enterprises in China. This phenomenon reflects that people in China are willing to spend their time and capital in sport, because of the interest or financial return. In a basic study, because of the interference of various factors such as athletes and sports clubs, financial returns from sports investments often do not yield satisfactory returns in short term and may even lose money. Although the benefits of sports investment are slow to emerge, once the benefits start to emerge, this will provide investors with long-term stable returns. As an investment that enhances the physical fitness of workers, the benefits of sports investment will play out over the lifetime of the worker. In addition, due to the hereditary and acquired nature of people's fitness, the improved fitness and health levels brought about by sports investment will be passed on to the next generation. In this way, it will be passed on from generation to generation, giving people long-term benefits. Therefore, sports investment must be strategic [2]. The financial return of an investment in sport industry is not usually obvious in short run, this is because both a team and a player have to grow their various abilities in this field in order to win games in the future. Therefore, the improvement will not be obvious in the short term. As a result, the profits of this investment will increase with the investee's growth in the competitive level. However, if players and teams move in unexpected directions, they would become uncertain, and match results and public opinion could lead to uncertain financial returns as well. In reality, there are also other factors, which can influence economic performance. The performance of business organizations is affected by their strategies and operations [3]. As suitable portfolio can make profits for investors, people can consider different stocks to purchase, so that they are possible to get higher interest and face lower risk. What is more, these kinds of methods can be built by operations after using statistical model.

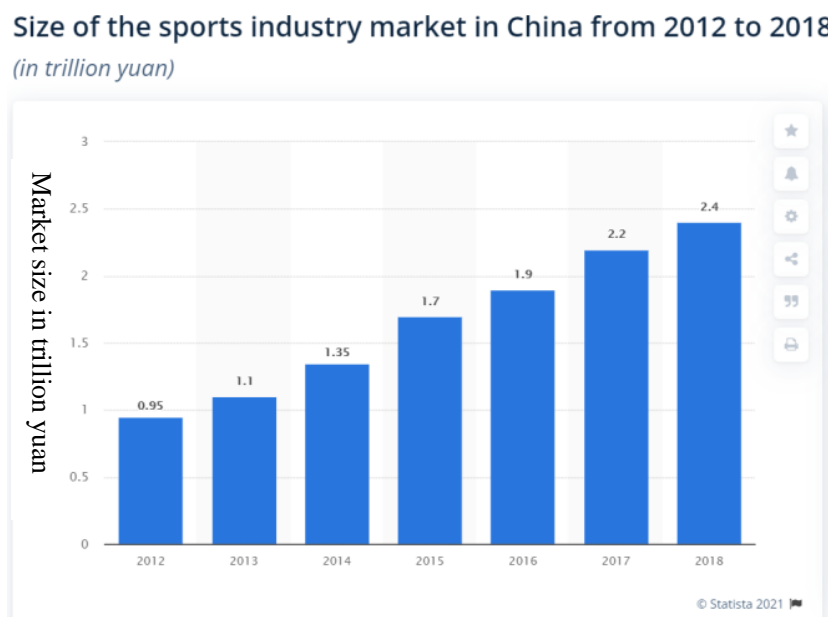

Figure 3 Size of the sport industry market in China from 2012 to 2018 [13]. 


\section{ANALYSIS OF RISKS OF INVESTMENT}

To illustrate the strategies, this article will analyze the risks of investment in sport industry. Basically, risks can be divided into five kinds, including policy, capital, market, brand and environment [4]. Policy risks from the government, which are always of a public interest nature, as the sports market is not normally strong enough to influence politics. The capital risk means that in the beginning of the investment in sport industry, the capital that investors need is large, thus they face obvious funding pressure. When the investment cannot be profitable in the first time, it is difficult for some firms to maintain their financial health. The reason is that they are necessary to spend capital for maintaining the normal operation of their company. Market risk refers to the risk that whether the investment project product or service is accepted and recognized by the market or users, thereby causing losses to the enterprise. Also, there are serious competition between the same occupations, and if they choose a similar market, the condition would be worse. Brand risk is that "The competition in the future market is the competition of corporate brands, and corporate branding is the core competitiveness of enterprises. A brand is composed of a certain unique knowledge, skills and experience in a certain environment. It is deposited in the corporate culture. It is a concentrated expression of inimitable corporate capabilities and requires long-term accumulation" [4]. The brand culture is hard to imitate because a brand includes the precipitation of time, indicating that it is also difficile for a firm to establish a brand culture. Moreover, the environmental risk can become a trouble as well. For example, when financial crisis happens, the market would change dramatically, so citizens need to pay more attention on their living standard, willing to purchase living goods rather than sports consumption. In China, especially before 2000, people's life changed based on the reform of government. However, this change is slight in short time, which means the new things are adopted by people tardily [4]. In a word, the risks above are necessary for investors to care seriously in order to build a suitable strategy.

Overall, the control of risk is one of the most important parts of investment, and the expansion of the sports market today is unstoppable, regardless of the economic stability of the market, according to the PwC sports data report [5]. Investing requires a discerning eye as well as caution to face and prevent various risks. Policy risk is the most immediate of these risks, as it is a matter of investment direction and strategy, which will be analyzed in the following section on investment strategies. There are also other risks that need to be taken into account, but they do not have the same direct impact on investment as policy. The movement of the macroeconomic markets is regulated by macroeconomic factors and policy changes are part of this. Changes in policy are more like trends. Going against the base composition of society often has unpredictable effects

\section{THREE STRATEGIES OF SPORT INVESTMENT}

\subsection{Developing a Strategy Based on Policy}

The first method is to Invest according to realtime policies which enacted in the National Government. Different countries have their own policies in sports, and the main common point is the positive policies published for development of sports. For instance, China's sports policy has been formulated with the objective of promoting the development of the sports industry, according to the policy published by "Twelfth Five-Year Plan for Sports Development", "to improve the level of public sports services, promote the development of mass sports, consolidate the international competitiveness of competitive sports and enhance the innovation capacity of the sports industry" [6]. Meanwhile, The State Council in China has also spoken out in 2014 about accelerating the development of the sports industry and increasing the amount of money invested to expand it. "We should accelerate the overall layout of the sports system, enrich sports products and services, further meet and stimulate the demand for sports consumption among the population. By 2035, it is expected to build a leading sporting nation" [7]. These policies are adopted by the government and international sports organizations. Thus, the scale of the sports industry has expanded rapidly. As a result, the policy makes the sports industry become an area which worth to investing in.

\subsection{Strategy Depending on Sporting Events}

The second method is to follow up the sport events, as they have great influence in the world and the large sport events attract every country's civilians. To study this method, the most important thing is defined the size of a sport competition, also, this study will target individual countries. The sport 
competitions can be divided into two types easily, which are large and small. The large games can be defined as the games that have international influence. In the meanwhile, the small games can be defined as the games that are normal but global. It is regular to see a large amount of people betting on sports currently. When the sport game starts, people guess which side will win, and they will invest the capital in the sport market, which belongs to the small match condition. Although it is the small scale, it influences many individuals as the same time in a market. For large games, "Further, we find no evidence supporting that industries, that a priori were more likely to extract direct benefits from the event, observe positive significant effects. Yet, when we control for anticipation, the stock price reactions around the announcements are significant" [8]. In their research, the Summer and Winter Olympic Games, the World Football Cup, the European Football Cup are studied. All of these games have great social influence. In normal, these kinds of games did not influence a country's stock market deeply. However, if the expectation has been controlled, the result will be a contradiction. In greater detail, major sporting event sponsorships have a huge impact on brands and brand equity power, according to a research [9].

\subsection{Indirect Investment Strategy}

The third method is to not invest directly in sport market, because the sports industry nowadays is relevant to a variety of fields. For example, the leisure industry, which sells sport equipment, "At present, Chinese enterprises do not have the ability to compete with foreign large enterprises in terms of product development and brand awareness. After China's accession to the WTO, the competitiveness of international brand-name products will be further strengthened." [10] It is a rare opportunity for investing sports leisure firm, like Lining and ANTA sport. Concluding in the chart (see "Figure 4") of ANTA sport annual financial Income Statement, which ANTA Sports' annual earnings are rising annually, in this economic condition investment in ANTA is more stable. The other orientation is that shift strategic targets to the hot media industry of today. According to the 40th Statistical Report on the Development of the Internet in China, as of June 2017, the size of China's Internet users had reached 7.51 billion, reaching the highest Internet penetration rate in the world. "On June 21, the 2018 China Media Industry Development Report was released in Beijing. According to "Figure 5", the total scale of China's media industry reached RMB
1.89 trillion in 2017, an increase of $16.6 \%$ compared with the previous year" [11]. As the news media industry has an anticipated future, in the area of sports, Tencent is a representative example, whose annual revenues have realized the gradual improvement in recent years. Between 2018 and 2019, the total revenues increased $12 \%$, the profits for a year increased $18 \%$, and share premium increased $23 \%$, although the trade war between the United States and China is still very scorching in 2019.

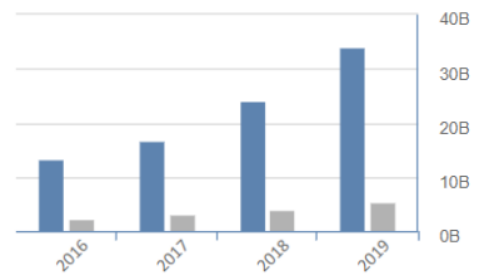

Figure 4 ANTA's report [14]. 


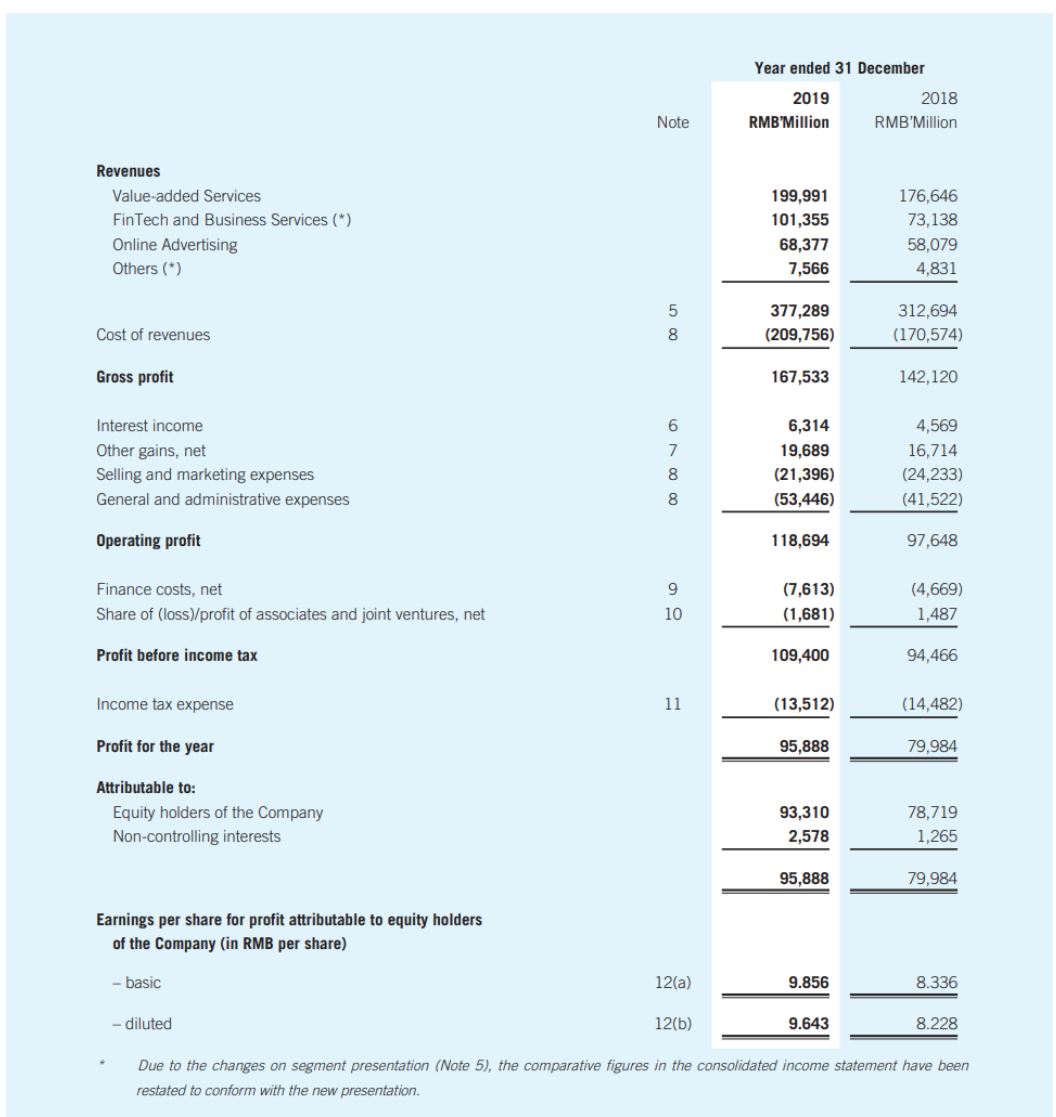

Figure 5 Tencent's report, Consolidated Income Statement [15].

As a result, the media market is potential around last decade. "Tencent has a strong advantage over other live sports video platforms in terms of attracting audiences and importing traffic, and has a large number of users" [12]. Tencent operates WeChat, QQ and Tencent video. Recent years, Tencent Sports has acquired the exclusive network broadcasting rights in China for three professional sports leagues, including the NBA (U.S. National Basketball Association), the NHL (National Hockey League) and the NFL (National Football League), and negotiations with MLB (Major League Baseball) underway [12]. Due to the data's approximation, Tencent Sports broadcasted over 1,500 NBA regular season games and playoff games in a single season. All of these conditions make it possible that Tencent can achieve profit growth for several years in a row.

\section{DISCUSSION}

These three strategies have limitations, but it is necessary and practical to adjust portfolio and investment strategy according to national policies. Each country has different policies, and going against the policy is Unworthy. Paying attention to the policy and market trends of the changes is the most important thing for investment. For the second strategy, it is theoretically feasible because major international sports events can bring a social stir. Both the media and the sports industry and various related fields are driven by major sports events, although the changes involved are not necessarily positive. However, according to the quote above, studies show no evidence that major sporting events could lead to large profits on investments in sports when not expected. For individuals, it may be profitable to bet on smaller games or buy stock in a club. Personal experience, such as familiarity with a particular team or a unique understanding of the game, is often more important in such investments. For the third investment strategy, generally speaking, in the Chinese market indirect investments are very practical. The public is interested in buying some sporting goods and fans are willing to pay to watch live matches on video software. Undoubtedly, live streaming software is not limited to Tencent Video. 


\section{CONCLUSION}

In the current sports market, although there are still many uncertainties and various investment risks, it is of utmost importance to develop different investment plans according to the different investment directions, investment targets and investment types. There are three investment strategies in this article which can give investors a direction to invest in, but these strategies cannot be generalized to all investments. In the market it is more a matter of statistical modelling and analyzing years of investment market data to construct portfolios. Overall, investing in the sports market is a hopeful investment direction and investing in the sports industry provides stable returns. With the strong government support, there will be more opportunities for investment in the sports industry.

\section{AUTHORS' CONTRIBUTIONS}

This article is independently completed by Yuhao Wang.

\section{ACKNOWLEDGMENTS}

First and foremost, I would like to show my deepest gratitude to my teachers and professors, who have provided me with valuable guidance in every stage of the writing of this thesis. Further, I would like to thank my friends and parents for their encouragement and support. Without all their enlightening instruction and impressive kindness, I could not have completed my thesis.

\section{REFERENCES}

[1] C. H. Zhang, 2000, The trend of globalization of the sports industry and its impact on the development of China's sports industry.

[2] Z. M. Lin, Yafei Wang, 1994, On sports investment and economic benefits, pp.77-78.

[3] Baron, D. P. 2000 Business and its environment, 3rd edn. Upper Saddle River, NJ: Prentice Hall

[4] Y. Wang, H. J. Li, An Analysis of the Investment Risk Factors in Recreation Sports Industry and Control Risk

[5] PwC, 2015, Changing the game, Outlook for the global sports market to 2015 .

[6] Twelfth Five-Year Plan for Sports Development, 2011
[7] Z. W. Gou, 2020, Speeding up the construction of sports power and creating a new situation of sports development, Study Times, vol. 1552, November $16^{\text {th }}, 2020$

[8] A. M. Martins, A. P. Serra, 2011, Market Impact of International Sporting and Cultural Events.

[9] Z. M. Liu, 2010, Research on the impact of major sporting event sponsorship on brands.

[10] W. G. Fu, 2008, Sports IndustrializationCorporate sports market investment strategy.

[11] 40th Statistical Report on the Development of the Internet in China, 2017, online available, accessed on March 20th, 2021, http://www.people.com.cn/.

[12] D. L. Guo, 2021, Present Situation and Development Strategy of Tencent Sports APP Live Sports.

[13] Size of the sports industry market in China from 2012 to 2018, online available, accessed on March 20th, 2021, https://www.statista.com/statistics/1131120/ch ina-sports-industry-market-size/

[14] 2020 Financial Summary, online available, accessed on March 20th, 2021, https://cn.investing.com/equities/anta-sportsfinancial-summary

[15] 2019 Annual report, online available, accessed on March 20th, 2021, https://static.www.tencent.com/uploads/2020/ 04/02/ed18b0a8465d8bb733e338a1abe76b73. pdf 